Vol. XX, No. 1

\title{
Twentieth Annual Saskatchewan Christmas Bird Count, 1961
}

\author{
Edited by Mary Houston, Saskatoon
}

The Regina observers organized by Frank Brazier set an all-time provincial record this year, sighting 37 species on December 24, 1961. They also added the only new species a Gadwall - to the 105 species previously observed in Saskatchewan during twenty years of Christmas counts. They sighted a Townsend's Solitaire for the second successive year.

Saskatoon set a record for the number of observers - 37 people braved a " 40 below zero wind chill temperature."

Snowy Owls were uncommon for the fourth successive year, being seen in only three localities. No Goshawks were seen at all! A total of 58 species was seen this year.

BATTLEFORD, Sask. Dec. $26 ; 30$ miles by car and 4 miles on foot in $51 / 2$ hours; temp. $-16^{\circ}$; wind N.W. at 15 mph.; 18 inches of snow. 14 species, 259 individuals. Ruffed Grouse, 11; Sharp-tailed Grouse, 87; Ring-necked Pheasant, 2; Rock Dove, 77; Great Horned Owl, 1; Hairy Woodpecker, 1; Downy Woodpecker, 1; Black-billed Magpie, 7; Blackcapped Chickadee, 11; Bohemian Waxwing, 54; Starling, 1; Evening Grosbeak, 3; Pine Grosbeak, 1; Snow Bunting, 2.-Spencer Sealy.

BLADWORTH, Sask. Jan. 1; 28 miles in 2 hours by vehicle in the a.m., $3 \frac{1}{2}$ hours by vehicle and on foot in the p.m.; temp. $20^{\circ}$ to $35^{\circ}$; wind W. at 10 mph.; clear. 12 species, 224 individuals. Sharp-tailed Grouse, 12; Gray Patridge, 28; Rock Dove, 3; Great Horned Owl, 2; Horned Lark, 17; Blue Jay, 1; Black-billed Magpie, 15; Black-capped Chickadee, 3; House Sparrow, 80; Pine Grosbeak, 2; Common Redpoll, 49; Snow Bunting, 12. (Add: Starling, 2. Dec. 31).-P. Lawrence Beckie.

BROADVIEW, Sask. Dec. 24; 64 miles by car in 4 hours; temp. $30^{\circ}$; calm, clear, slight haze. 6 species, 148 individuals. Sharp-tailed Grouse, 6; Great Horned Owl, 1; Black-billed
Magpie, 5; Black-capped Chickadee, 1; House Sparrow, 35; Common Redpoll, 100. (Add: Ring-necked Pheasant, 4, Jan. 1; Gray Partridge, 5, Dec. 30; Rock Dove, 3, Dec. 25; Evening Grosbeak, 12, Dec. 29; Pine Grosbeak, 5, Dec. 26; Snow Bunting, 200, Dec. 25).-Adrey and Charles Thacker.

BUFFALO POUND LAKE, Sask. Dec. $30 ; 10$ miles by car and 14 miles on foot in 6 hours; temp. $15^{\circ}$; wind N.W. $15 \mathrm{mph}$; light fog, clearing later; 8 inches of snow. 5 species, 41 individuals. Gray Partridge, 19; Great Horned Owl, 1; Black-billed Magpie, 1; Black-capped Chickadee, 1; Pine Grosbeak, 19.-Michael Rhodes, George Rhodes, John Horton, Carl Ellis (compiler).

CARON, Sask. Dec. $31 ; 10$ miles by car in $1 \frac{1 / 2}{2}$ hours; temp. $20^{\circ}$; partly clouded; wind $15 \mathrm{mph}$. increasing to $25 \mathrm{mph}$. 6 species, 105 individuals. Sharp-tailed Grouse, 14; Gray Partridge, 7; Black-billed Magpie, 9; House Sparrow, 30; Common Redpoll, 25; Snow Bunting, 20.-Mr. and Mrs. Cy Knight.

CRAVEN, Sask. Dec. 29; 50 party miles by car, and 82 party miles on foot in $8 \frac{1}{2}$ hours; temp. $-10^{\circ}$ to $-2^{\circ}$; wind S.E. $36 \mathrm{mph}$. to $12 \mathrm{mph}$.; snow cover light in the open, 18 inches deep in the brush. 22 species, 1517 individuals. Golden Eagle, 1; Bald Eagle, 1; Prairie Falcon, 1; Ruffed Grouse, 2; Sharp - tailed Grouse, 1; Ring-necked Pheasant, 2; Gray Partridge, 8; Rock Dove, 12; Great Horned Owl, 5; Short-eared Owl, 1; Hairy Woodpecker, 3; Downy Woodpecker, 5; Black-billed Magpie, 74; Black-capped Chickadee, 32; Bohemian Waxwing, 40; Starling, 1; House Sparrow, 1,000; Evening Grosbeak, 4; Pine Grosbeak, 33; Common Redpoll, 254; Song Sparrow, 1; Snow Bunting, 36.-G. Anweiler, F. Brazier, W. Fleming, E. Fox (compiler), R. Fox, R. Lein, B. Nelson, R. W. Nero, R. Sanderson, A. Wade, D. Wade. 
DILKE, Sask. Dec. 24; 50 miles by car and on foot in $21 / 2$ hours over rolling prairie farmland with frequent blufs, farm shelterbelt and Arm River Valley; temp. $34^{\circ}$; wind S.W. at 15-25 mph.; snow 10 inches, drifted. 13 species, 628 individuals. Prairie Falcon, 1; Sharp-tailed Grouse, 9; Ring-necked Pheasant, 1; Gray Partridge, 30; Rock Dove, 1; Horned Lark, 12; Black-billed Magpie, 12; Blàck-capped Chickadee, 3; Starling, 7; House Sparrow, 150; Pine Grosbeak, 3; Common Redpoll, 200; Snow Bunting, 200.-J. B. Belcher, Margaret Belcher.

DUBUC, Sask. Dec. 27; 5 miles on foot. 10 species, 125 individuals. Gray Partridge, 15; Hairy Woodpecker, 1; Downy Woodpecker, 1; Black-billed Magpie, 3; Black-capped Chickadee, 6; Bohemian Waxwing, 15; House Sparrow, 75; Pine Grosbeak, 4; Slate-colored Junco, 1; Snow Bunting, 4. (Add: Ruffed Grouse, 1; Dec. 28; Sharp-tailed Grouse, 6, Dec. 21; Starling, 13, Dec. 21).-George Chopping.

ESTEVAN, Sask. Dec. $31 ; 4$ miles on foot and 40 miles by car in $53 / 4$ hours; temp. $15^{\circ}$; wind S.W. 10-15 mph.; light snow falling; 8 inches of snow on ground. 12 species, 465 individuals. Mallard, 15; Pintail, 1; Lesser Scaup, 1; Downy Woodpecker, 1; Black-billed Magpie, 10; Blackcapped Chickadee, 16; Whitebreasted Nuthatch, 1; Bohemian Waxwing, 4; Starling, 1; House Sparrow, 385; Pine Grosbeak, 29; Common Redpoll, 1. (Add: Gray Partridge, 4, Dec. 24; Horned Lark, 2, Jan. 1; Snow Bunting, 5, Jan. 1).-Darrel Carlson, Ross Lein (compiler).

FORT QU'APPELLE, Sask. Dec. 23. 9 species, 242 individuals. Ruffed Grouse, 1; Rock Dove, 1; Downy Woodpecker, 2; Black-billed Magpie, 6; Black-capped Chickadee, 11; Bohemian Waxwing, 50; House Sparrow, 150; Pine Grosbeak, 9; Snow Bunting, 12. (Add: Great Horned Owl, 1, Dec. 30; Hairy Woodpecker, 1; Horned Lark, 2, Dec. 31; Blue Jay, 1; Northern Shrike, 1; Evening Grosbeak, 1; Common Redpoll, 15; Slatecolored Junco, 2).-Alden Barnett, Dr. G. D. Barnett, Nancy Bushell, E. M. Callin (compiler), Richard Carter, Errol Cochrane, Dr. H. D.
Jenner, Richard Nevard, Horace Reed, Jos. Rumancik.

GRENFELL, Sask. Dec. 25; 10 miles by truck and on foot about the farm; temp. $7^{\circ}$ to $-5^{\circ}$; wind N.W. 20-25 mph.; cloudy; 6 inches of snow and bare fields; parkland. 6 species, 147 individuals. Black-billed Magpie, 5; Black-capped Chickadee, 1; Bohemian Waxwing, 30; House Sparrow, 75; Pine Grosbeak, 1; Snow Bunting, 35. (Add: Sharp-tailed Grouse, 15, Dec. 23; Gray Partridge, 9, Dec. 31; Hairy Woodpecker, 1, Dec. 31 ; Common Redpoll, 32, Dec. 31).-Mr. and Mrs. John Hubbard.

HAWARDEN, Sask. Dec. 28; 20 miles by car and on foot around farm in 5 hours; temp. $-2^{\circ} ; 12$ inches of snow; open prairies, farm yard and groves of trees. 4 species, 126 individuals. Gray Partidge, 21; Black-billed Magpie, 2; House Sparrow, 100; Snow Bunting, 3. (A d d : Sharp-tailed Grouse, 1, Dec. 30; Rock Dove, 10, Dec. 31; Snowy Owl, 2, Dec. 31; Common Redpoll, 1, Dec. 30 and Jan. 1). -Harold Kvinge.

HIGH HILL-KLOGEI LAKE, Sask. Dec. $31 ; 14$ miles on foot through heavy bush and poplar bluffs in 6 hours; temp. $-5^{\circ}$; clear, clouding in late afternoon. 10 species, 26 individuals. Golden Eagle, 1; Sharp-tailed Grouse, 1; Pileated Woodpecker, 1; Hairy Woodpecker, 2; Black-backed Three-toed Woodpecker, 1; Gray Jay, 3; Blue Jay, 1; Black-billed Magpie, 6; Raven, 7; Black-capped Chickadee, 3.-Anton Waycheshen.

KELVINGTON, Sask. Jan. 1; within Kelvington town limits in 2 hours; temp. $26^{\circ}$; no wind; foggy. 5 species, 164 individuals. Black-capped Chickadee, 5; White-breasted Nuthatch, 1; Bohemian Waxwing, 3; House Sparrow, 150; Evening Grosbeak, 5. (Add: Ruffed Grouse, 3, Dec. 23; Blue Jay, 2, Dec. 23; Starling, 1, Dec. 21).Steve and Naomi Waycheshen.

LADY LAKE, Sask. Dec 28; 5 hours on foot; temp. $-10^{\circ}$ to $-15^{\circ}$; wind light; clear. 9 species, 112 individuals. Ruffed Grouse, 1; Gray Partridge, 1; Rock Dove, 10; Downy Woodpecker, 1; Gray Jay, 3; Black-billed Magpie, 2; Black - capped Chickadee, 18; White-breasted Nuthatch, 1; House Sparrow, 75. (Add: Hairy Wood- 
pecker, 1, Dec. 30).—Donald J. Buckle.

LEADER, Sask. Dec. 27; 3 hours on foot and 1 hour and 24 miles by car; temp. $0^{\circ}$; no wind; foggy; 10 inches of snow. 5 species, 173 individuals. Ring-necked Pheasant, 9; Gray Partridge, 34, Rock Dove, 3; Black-billed Magpie, 13; House Sparrow, 114. (Add: Sharp-tailed Grouse, 10, Dec. 30).-Daisy D. Myers.

MASEFIELD, Sask. Dec. 25; 61/2 miles by foot along the Frenchman River and 42 miles by car; temp. $28^{\circ}$; sunny, clouding over; 5 inches of snow. 10 species, 619 individuals. Golden Eagle, 1; Ring-necked Pheasant, 8; Gray Partridge, 34; Hawk Owl, 1 (no details, Ed.); Black-billed Magpie, 15; Black-capped Chickadee, 8; House Sparrow, 400; (Pine?) Grosbeak, 2; Common Redpoll, 100; Snow Bunting, 50. (Add: Horned Lark, 1, Dec. 24).-J. David Chandler.

MELVILLE, Sask. Dec 24; 20 miles by car and 5 miles on foot in 4 hours; light south wind; cloudy; 8 inches of snow. 11 species, 284 individuals. Ruffed Grouse, 1; Hairy Woodpecker, 1; Horned Lark, 6; Blue Jay, 5; Black-billed Magpie, 9; Black-capped Chickadee, 9; House Sparrow, 150, Evening Grosbeak, 3; Pine Grosbeak, 4; Common Redpoll, 95. (Add: Bohemian Waxwing, 3, Dec. 21; Common Grackle, 1, Dec. 19). - Gary Anweiler.

MOOSE JAW, Sask. Dec. 26; 34 party miles by car and 14 by foot in 29 party hours; temp. $-9^{\circ}$ to $-7^{\circ}$; wind N.W. at $10 \mathrm{mph}$. gusting to $20 ; 5$ inches of snow. 16 species, 718 individuals. Prairie Falcon, 1; Ringnecked Pheasant, 16; Gray Partridge, 32; Rock Dove, 100; Snowy Owl, 1; Downy Woodpecker, 1; Black-billed Magpie, 61; Common Crow, 4; Blackcapped Chickadee, 2; Robin 1; Bohemian Waxwing, 105; House Sparrow, 350; Evening Grosbeak, 1; Pine Grosbeak, 20; Common Redpoll, 3; Snow Bunting, 20. (Add: Great Horned Owl, 1, Dec. 28).-Frank Hill, Carl Ellis, w. W. Riome (compiler), Robert Tabaka, Mrs. F. V. Humphreys, Mrs. A. Pugh, Mrs. R. J. Dunn, Mrs. D. Rhodes, George Rhodes, Michael Rhodes, Jack Zess, John Horton, Mary Jane Ellis (members of Moose Jaw Natural History Society).
REGINA, Sask. Dec. $24 ; 137$ car miles and 48 man miles; temp. $11^{\circ}$ to $35^{\circ}$; wind S.E. 16 mph.; bright sunshine 2.4 hours. 37 species, 5475 individuals. Horned Grebe, 1; Pied-billed Grebe, 1; Mute Swan, 5; Whistling Swan, 2; Canada Goose, 100; Mallard, 300; Gadwall, 2; Lesser Scaup, 12; Common Goldeneye, 9; Ruddy Duck, 6; Common Merganser, 1; Sharp-tailed Grouse, 7; Ring-necked Pheasant, 42; Gray Partridge, 71; American Coot, 3; Rock Dove, 119; Great Horned Owl, 5; Snowy Owl, 5; Yellowshafted Flicker, 1; Downy Woodpecker, 4; Horned Lark, 1; Blackbilled Magpie, 31; Black-capped Chickadee, '; Red-breasted Nuthatch, 3; Robin, 1; Townsend's Solitaire, 1; Bohemian Waxwing, 757; \&orthern Shrike, 1; Starling, 5; House Sparrow, 3,815; Common Grackle, 2; Pine Grosbeak, 114; Common Redpoll, 3; Slate-colored Junco, 3; Harris' Sparrow, 1; Song Sparrow, 1; Snow Bunting, 10. (Add: White-breasted Nuthatch, 1, Dec. 30 and Jan. 1; Evening Grosbeak, 1).-Mr. and Mrs. Doug Wade, Alan Wade, Vic Wilshire, Elmer Fox, Reg Fox, Billy Fieming, Ricky Sanderson, Dave Adams, Doug Gilroy, Jessie Bailey, Ruth and Herb Tempel, Elsie Cheseman, George Ledingham, Maureen Rever, Ruth Bennett, Ron Austin, Frank Brazier (compiler), Bob Nero, Robert McCall, Howard Erickson, Walter Knutson.

SASKATOON, Sask, Dec. $26 ; 22$ party miles. on foot in 17 party hours and $191 \frac{1}{2}$ party miles by car in 21 party hours; temp. $-9^{\circ}$ to $-13^{\circ}$; wind W.N.W. 13 to $23 \mathrm{mph}$.; partly cloudy; 5 inches of snow. 19 species, 3,619 individuals. Common Goldeneye, 3; Pigeon Hawk, 2; Sharp-tailed Grouse, 41; Ring-necked Pheasant, 3; Gray Partridge, 42; Rock Dove, 164; Snowy Owl, 2; Downy Woodpecker, 1; Blue Jay, 1; Black-billed Magpie, 85; Black-capped Chickadee, 25; Robin, 1 ; Bohemian Waxwing, 663; Northern Shrike, 1; House Sparrow, 1791; Evening Grosbeak, 12; Pine Grosbeak, 54; Common Redpoll, 556; Snow Bunting, 172. (Add: Mallard, 5).-Jay Beaman, Harold Belcher, Al Binnie, John Black, Pern Cordery, Murray Cox, Dr. and Mrs. Ray Denson, Mr. and Mrs. Hans Dommasch, Bob Folker, George Gerrity, Mr. and Mrs. Bernard Gollop, Michael Gollop, Vic Harper, Mr. and Mrs. 
Jim Hogg, Vern Irvin, Stanley Houston, Dr. and Mrs. Stuart Houston, Dr. C. J. L'Ami, Jean Mackenzie, Janet McDougall, Sigrid Martynes, Bob Mills, Dr. and Mrs. Jim Pepper, Frank Roy (compiler), Margaret Sawatzky, John Shadick, Dorothy Slater, John Van Mossel, Peter Walmsley, Ted Wedge, Terry Wedge (members and friends of the Saskatoon Natural History Socièty).

SHAUNAVON, Sask. Jan $1 ; 31 / 2$ miles on foot along White Mud River, 60 miles by car; temp. $40^{\circ}$; sunny; 3 inches of snow. 10 species, 359 individuals. Golden Eagle, 1; Ruffed Grouse, 10; Sage Grouse, 11; Ringnecked Pheasant, 1; Gray Partridge, 9; Snowy Owl, 1; Long-eared Owl, 1; Horned Lark, 14; Black-billed Magpie, 13; House Sparrow, 300.-James Stephenson, Doug E. Young (compiler).

SKULL CREEK, Sask. Jan. $6 ; 21 / 2$ hours; temp. $34^{\circ}$ to $28^{\circ}$; wind light; clear. 17 species, 432 individuals. Rough-legged Hawk, 1; Prairie Falcon, 1; Sharp-tailed Grouse, 21; Ringnecked Pheasant, 18; Gray Partridge, 48; Long-eared Owl, 6; Hairy Woodpecker, 2; Downy Woodpecker, 1; Horned Lark, 12; Blue Jay, 1; Blackbilled Magpie, 65; Black-capped Chickadee, 41; Northern Shrike, 1; House Sparrow, 192; Pine Grosbeak, 1; Common Redpoll, 20; Tree Sparrow, 1. (Add: Golden Eagle, 2, Dec. 30 and Jan. 1; Short-eared Owl, 1, Jan. 1; Gray Jay, 1, Jan. 4; Snow Bunting, 550, Jan. 4).-Mr. and Mrs. Borman, George, Peter and Donna Swain, Joan Stevens, Stanley Birchall, Harry Williams, S. A. Mann (compiler), Mrs. S. A. Mann, Robert Mann.

SPIRIT LAKE, Sask. Dec 31; 4 hours and 5 miles on foot; temp. $14^{\circ}$; wind l ight; intermittently cloudy. 13 species, 165 individuals. Ruffed Grouse, 3; Hairy Woodpecker, 6; Downy Woodpecker, 7; Blue 'Jay, 1; Black-billed Magpie, 4; Black-capped Chickadee, 65; White-breasted Nuthatch, 2; Bohemian Waxwing, 4; House Sparrow, 35; Evening Grosbeak, 5; Pine Grosbeak, 2; Common Redpoll, 19; Snow Bunting, 12; (Add: Sharp-tailed Grouse, 17, Jan. 1).Joyce Gunn, Bill Anaka.

STAR CITY, Sask. Dec. 23; 3 miles on foot in 4 hours; temp. $-5^{\circ}$; wind light from S.S.E.; clear; 18 inches of snow. 4 species, 48 individuals. Black-billed Magpie, 1; Black-capped Chickadee, 1; House Sparrow, 40; Evening Grosbeak, 6. (Add:'Sharptailed Grouse, 14, Dec. 24; Downy Woodpecker, 2, Dec. 30; Bohemian Waxwing, 1, Dec. 30).-David and Wesley Grout.

WOODROW, Sask. Dec. 27; 30 miles by truck and 2 miles on foot in 4 hours; temp. $-6^{\circ}$ to $2^{\circ}$; wind calm to N.W. at $15 \mathrm{mph}$.; treeless prairie with 22 farm groves-roads bare, about 1 foot of snow in groves. 7 species, 1413 individuals. Ringnecked Pheasant, 26; Gray Partridge, 64; Rock Dove, 8; Horned Lark, 2; Black-billed Magpie, 5; House Sparrow, 1300; Snow Bunting, 8.-Fred Parchman.

OAK LAKE, Man. Dec. 30; 6 miles on foot in 4 hours, and 46 miles by car in $4 \frac{1}{2}$ hours; temp. $-5^{\circ}$ to $19^{\circ}$; wind 10 to $17 \mathrm{mph}$. shifting from south to northwest; snow 4 inches deep. 16 species, 1099 individuals. $\mathrm{R}$ uffed Grouse, 1; Sharp-tailed Grouse, 21; Short-eared Owl, 1; Downy Woodpecker, 5; Horned Lark, 7; Blue Jay, 2; Black-billed Magpie, 19; Black-capped Chickadee, 36; White-breasted Nuthatch, 7; Robin, 2; House Sparrow, 290; Evening Grosbeak, 2; Pine Grosbeak, 9; Hoary Redpoll, 12; Common Redpoll, 443; Snow Bunting, 242. (Add: Great Horned Owl, 1, Dec. 23; Snowy Owl, 1, Dec. 28).-David R. M. Hatch.

\section{CO-OPERATIVE SPRING MIGRATION STUDY}

Once again a continent-wide survey is being made under the auspices of the U.S. Fish and Wildlife Service of spring migration for a selected species (for list of species see Blue Jay, March, 1961 , or write to the address below). Readers of the Blue Jay are urged to keep spring migration dates and submit their records by June 15, 1962, to Mrs. Dorothy Wade, 1351 Jubilee Ave., Regina. 